\title{
Effect of Sinusoidal Thickness Variation on Vibrations of Non-homogeneous Parallelogram Plate with Bi-linearly Temperature Variations
}

\author{
Anupam Khanna* and Pratibha Arora \\ Department of Mathematics, M. M. Engineering College, M. M. University, \\ Mullana, Haryana, India; rajieanupam@gmail.com, pratibha360@gmail.com
}

\begin{abstract}
The present study is the computational prediction about the effect of sinusoidal varying thickness on the vibrations of non-homogeneous parallelogram plate. Consideration of the plate's material is visco-elastic with clamped boundary. It is assumed that the temperature varies bi-linearly while the density of the plate's material varies linearly in one direction due to non-homogeneity. The general equation of motion and consecutive equations are solved by using the Rayleigh-Ritz method. To study the vibrational behavior of parallelogram plate, natural frequency for both the modes of vibration is calculated for different values of parameters and shown in the graphs. All the calculations are carried out for duralumin.
\end{abstract}

Keywords: Vibration, Taper Constant, Sinusoidal, Thickness Variation, Non-homogeneity, Frequency, Thermal Gradient.

\section{Motivation}

The theory of thermo elasticity deals with the effect of thermal disturbance on elastic bodies. This theory is a subject of considerable interest from both points of view concerning the formulation of mechanical as well as a mathematical point of view. The importance of study of the effect of temperature on vibration was recognized in the $19^{\text {th }}$ century and mathematical models have been developed to describe the effect of temperature on vibration of plates. The origin of such studies or theories was stated by Leissa in 1967. In recent years, owing to the diversification of engineering materials and for operation in several thermal environments i.e. nuclear weapons, missiles, defense weapons, laser weapons etc. thermal problems have become very important for modern designers and researchers. The classical theory of vibration (which deals with the effect of thermal gradient on vibration) has attracted the attention of many researchers because of its extensive use in diverse field. Authors analyzed the two dimensional temperature effect on the vibrations of non-homogeneous tapered parallelogram plate so that researchers and designers can take advantages from the findings of this paper and can develop more authentic and efficient structures or mechanical designs.

\section{Introduction}

The plates of variable thickness are frequently used as structural components and their vibration characteristics are important for practical design. The structural components are used in many applications involving aerospace, submarine structural, civil engineering structures etc. Depending upon the requirement, durability and reliability, materials are being developed so that these may provide better strength, efficiency and economy. Therefore a study of character and behavior of these plates is required so that the full potential of these plates may be used. These plates

* Corresponding author:

Anupam Khanna (rajieanupam@gmail.com) 
may be of any type for example-rectangular plate, square plate, triangular plate. Also, the thickness of these plates affects the behavior of plates.

Leissa $[12,13]$ mentioned some papers dealing with square, rectangular, parallelogram, circular, elliptic in his excellent survey. S. Chakraverty [2] has studied natural frequencies for free vibration of non-homogeneous elliptic and circular plates using two dimensional orthogonal polynomials. Singh and Sexena [1] has studied the transverse vibration of skew plates with variable thickness. Notable contributions are available on vibrations of rectangular with variable thickness. Tomar and Gupta [15] has studied the thermal effect of frequencies of an orthotropic rectangular plate of linearly varying thickness. In Roshan [11] has studied the effect of non-homogeneity on the vibration of orthotropic rectangular plates. Li [14] has done a vibrational analysis of rectangular plate with general elastic boundary support. Gupta [3, 7] has studied the effect of thermal gradient on visco-elastic rectangular plate of varying thickness. Khanna and Kumar [8] has studied thermally induced vibration of non-homogeneous viscoelastic plate of variable thickness. Khanna and Sharma [10] have made a vibration analysis of non-homogeneous square plate Khannna et al. [9] have studied the effect of varying Poisson ratio on thermally induced vibration of non-homogeneous rectangular plate. Gupta $[5,6]$ has studied the effect of thermal gradient on linearly varying in one direction and bi-direction, parabolic varying thickness of parallelogram plates, but none has done work on sinusoidal varying thicknesses of parallelogram plate. Therefore, aim of this paper is to study the effect of thermal gradient on non-homogeneous parallelogram plates with sinusoidal thickness variation in $\mathrm{x}$-direction. Rayleigh-Ritz technique is applied to get the numeric values of frequencies and results are shown graphically.

\section{Materials and Methods}

\subsection{Analysis and Equation of Motion}

A parallelogram plate $\mathrm{R}$ ( $\mathrm{a} \times \mathrm{b}$ ) with skew angle $(\theta)$ is shown in Figure 1. The skew plate is assumed to be non-uniform, thin and isotropic. The skew coordinates are related as

$$
\mathrm{x}=\mathrm{X}-\mathrm{Y} \tan \theta \text { and } \mathrm{y}=\mathrm{Y} \operatorname{Sec} \theta
$$

The boundaries of the plate in oblique coordinates are

$$
\mathrm{x}=0, \mathrm{x}=\mathrm{a} \text { and } \mathrm{y}=0, \mathrm{y}=\mathrm{b}
$$

For free vibrations of parallelogram plate, the displacement is assumed as [12]

$$
\mathrm{w}(\mathrm{x}, \mathrm{y}, \mathrm{t})=\mathrm{W}(\mathrm{x}, \mathrm{y}) \sin \omega \mathrm{t}
$$

where $\mathrm{W}(\mathrm{x}, \mathrm{y})$ is the maximum displacement at time $\mathrm{t}$ and $\omega$ is the angular frequency.

The maximum kinetic energy $\bar{T}$ and strain energy $\bar{V}$ at the plate when it is executing transverse vibration mode shape $\mathrm{W}(\mathrm{x}, \mathrm{y})$ as in [12] are

$$
\bar{T}=\frac{1}{2} \omega^{2} \cos \theta \iint h \rho W^{2} d x d y
$$

and

$$
\begin{aligned}
& \bar{V}=\frac{1}{2 \cos ^{3} \theta} \iint D\left[W,_{\left(x x^{2}\right)}-4 \sin \theta W,_{x x} W,_{x y+} 2\left(\sin ^{2} \theta+v \cos ^{2} \theta\right)\right. \\
& W,_{x x} W,_{y y}+2\left(1+\sin ^{2} \theta-v \cos ^{2} \theta\right) W,_{x y^{2}}-4 \sin \theta W,_{x y} W,_{y y}+ \\
& \left.W,_{y y^{2}}\right] d x d y
\end{aligned}
$$

A comma followed by a suffix denotes partial differential with respect to that variable.

Here $D=\frac{E h^{3}}{24\left(1-v^{2}\right)} \quad(6)$ is the flexural rigidity ( $v$ is Poission Ratio).

The modulus of elasticity $E$ which depends upon temperature $\tau$ is given by

$$
E(\tau)=E_{0}(1-\gamma \tau)
$$

where $\mathrm{E}_{0}$ is the Young modules at $\tau=0$

The thickness variation of the parallelogram plate is assumed to be sinusoidal in $\mathrm{x}$-direction (as shown in Figure 2) is

$$
h=h_{0}(1+\beta \sin [\pi x / 2 a]
$$

where $\beta$ is the taper constant in $\mathrm{x}$-direction and $h=h_{0}$ when $x=0$

Since plate is considered non-homogeneous, variation in the density of the plate is taken as:

$$
\rho=\rho_{0}\left(1-\alpha_{1}\left(\frac{x}{a}\right)\right)
$$

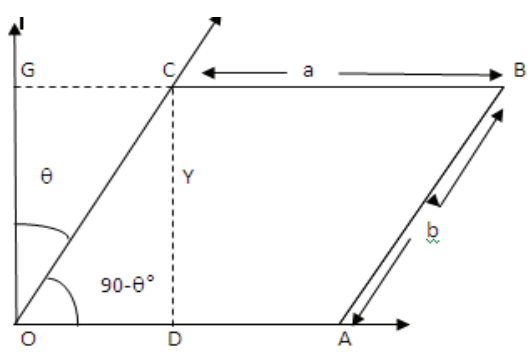

Figure 1. A parallelogram plate of lateral sides $a$ and $b$. 
Here $\rho_{0}$ is density at the center of plate and $\alpha_{1}$ is non homogeneity constant.

Assumed that plate is subjected to a study two dimensional linear temperature variations i.e. $x$-direction and $\mathrm{y}$-direction as

$$
\tau=\tau_{0}\left(1-\frac{x}{a}\right)\left(1-\frac{y}{b}\right)
$$

Here $\tau$ is the temperature excess above the reference temperature at any point $(\mathrm{x} / \mathrm{a}, \mathrm{y} / \mathrm{b})$ and $\tau_{0}$ at $\mathrm{x}=\mathrm{a}, \mathrm{y}=\mathrm{b}$. Using (10) in (7) one obtains

$$
E(x)=E_{0}\left(1-a\left(1-\frac{x}{a}\right)\left(1-\frac{y}{b}\right)\right)
$$

where $\alpha=\gamma \tau_{0}(0 \leq \alpha<1)$, is a parameter known as temperature gradient.

Using (6), (8), (9) and (11) in (4) and (5), one gets

$\bar{T}=\frac{1}{2} h_{0} \rho_{0} \omega^{2} \iint_{00}^{b a}\left(1+\beta \sin \left[\frac{\pi x}{2 a}\right]\right)\left(1-a_{1}\left(\frac{x}{a}\right)\right) W^{2} d x d y$

$\bar{V}=\frac{E_{0} h_{0}{ }^{3}}{24\left(1-v^{2}\right) \cos ^{4} \theta} \iint_{00}^{b a}\left(1-\alpha\left(1-\frac{x}{a}\right)\left(1-\frac{y}{b}\right)\right)(1+$

$\left.\beta \sin \left[\frac{\pi x}{2 a}\right]\right)^{3} \times\left[W,_{x^{2}}-4\left(\frac{a}{b}\right) \sin \theta W,_{x x} W,_{x y}+2\left(\frac{a}{b}\right)\left(\sin ^{2} \theta+\right.\right.$

$\left.\nu \cos ^{2} \theta\right) W,_{x x} W,_{y y}+2\left(\frac{a}{b}\right)\left(1+\sin ^{2} \theta-v \cos ^{2} \theta\right) W,_{x y^{2}}-$

$\left.4\left(\frac{a}{b}\right)^{3} \sin \theta W_{x y} W_{y y}+\left(\frac{a}{b}\right)^{4} W_{y_{y y^{2}}}\right] d x d y$

\subsection{Solution and Frequency Equation}

In Rayleigh-Ritz technique, maximum strain energy must be equal to the maximum kinetic energy. Hence, for arbitrary variations of $\mathrm{W}$ satisfying geometric boundary conditions, one finds that

$$
\delta(\bar{V}-\bar{T})=0
$$

For a parallelogram plate clamped along all the four edges, the boundary conditions are

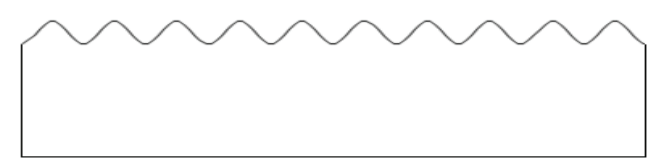

Figure 2. Sinusoidal thickness variation in a parallelogram plate.

$$
\mathrm{W}=\mathrm{W}, \mathrm{x}=0 \text { at } \mathrm{x}=0 \text {, a and } \mathrm{W}=\mathrm{W}, \mathrm{y}=0 \text { at } \mathrm{y}=0, \mathrm{~b}
$$

Corresponding two term deflection function is taken as [12]

$$
\begin{aligned}
\mathrm{W}(\mathrm{x}, \mathrm{y})= & \left(\frac{x^{2}}{a^{2}}\right)\left(\frac{y^{2}}{b^{2}}\right)\left(1-\frac{x}{a}\right)^{2}\left(1-\frac{y}{b}\right)^{2} \times\left[A_{1}+A_{2}\left(\frac{x}{a}\right)\left(\frac{y}{b}\right)\right. \\
& \left.\left(1-\frac{x}{a}\right)\left(1-\frac{y}{b}\right)\right]
\end{aligned}
$$

Using (12) and (13) in (14), one gets

$$
\delta\left(V_{1}-\lambda^{2} T_{1}\right)=0
$$

where

$V_{1}=\frac{1}{\left(\cos ^{4} \theta\right)} \iint_{00}^{b a}\left(1-\alpha\left(1-\frac{x}{a}\right)\left(1-\frac{y}{b}\right)\right)\left(1+\beta \operatorname{Sin}\left[\frac{\pi x}{2 a}\right]\right)^{3} \times$ $\left[W,_{\left(x x^{2}\right)}-4\left(\frac{a}{b}\right) \sin \theta W,_{x x} W,_{x y}+2\left(\frac{a}{b}\right)^{2}\left(\sin ^{2} \theta+v \cos ^{2} \theta\right)\right.$

$W,_{x x} W,_{y y}+2\left(\frac{a}{b}\right)^{2}\left(1+\sin ^{2} \theta-v \cos ^{2} \theta\right) W,_{x y^{2}}-4\left(\frac{a}{b}\right)^{3}$

$\left.\sin \theta W, A_{x y} W,_{y y}+\left(\frac{a}{b}\right)^{4} W,_{y y^{2}}\right] d x d y$

$T_{1}=\iint_{00}^{b a}\left(1+\beta \operatorname{Sin}\left[\frac{\pi x}{2 a}\right]\right)\left(1-a_{1}\left(\frac{x}{a}\right)\right) W^{2} d x d y$

and $\lambda^{2}=\frac{12 a^{4} \omega^{2} \rho_{0}\left(1-v^{2}\right)}{E_{0} h_{0}{ }^{2}}$ (a frequency parameter).

Equation (17) involves the unknown $A_{1}$ and $A_{2}$ arises due to the substitution of $\mathrm{W}(\mathrm{x}, \mathrm{y})$ from Equation (16). These unknowns are to be determined from Equation (17) for which

$$
\frac{\partial}{\partial A_{n}}\left(v_{1}-\lambda^{2} T_{1}\right)=0, n=1,2
$$

The above equation simplifies to

$$
b_{n 1} A_{1}+b_{n 2} A_{2}=0, n=1,2
$$

where $b_{n 1}, b_{n 2}(\mathrm{n}=1,2)$ involve parametric constants and frequency parameter.

For a non trivial solution the determinant of the coefficients of Equation (19) must be zero.

Therefore one gets the frequency equation as

$$
\left|\begin{array}{ll}
b_{11} & b_{12} \\
b_{21} & b_{22}
\end{array}\right|=0
$$

From Equation (20) one can obtain the quadratic equation in $\lambda^{2}$ from which two values of $\lambda^{2}$ can be found.

The frequency parameter $\lambda$ corresponding to first two modes of vibration of clamped parallelogram plate have been computed for various values of temperature gradient $(\alpha)$, non-homogeneity $\left(\alpha_{1}\right)$, skew angle $(\theta)$, aspect ratio $(\mathrm{a} / \mathrm{b})$ and taper constant $(\beta)$. The value of Poisson ratio $(v)$ is taken as 0.345 . The all results are shown graphically. 


\section{Results and Discussion}

To study the behavior of frequency, a comprehensive numerical computation for frequency parameter $\lambda$ corresponding to first two modes of vibration of parallelogram plate is carried out for various values of temperature gradient $(\alpha)$, non-homogeneity $\left(\alpha_{1}\right)$, skew angle $(\theta)$, aspect ratio $(\mathrm{a} / \mathrm{b})$, taper constant $(\beta)$.The value of Poisson ratio $v$ is taken 0.345 . The all results are shown graphically.

Graphs (1) and (2) are plotted for first two modes of frequencies respectively for variances $\beta$ from 0 to 1 with $\alpha=0$, $\alpha_{1}=0, \mathrm{a} / \mathrm{b}=1.5$ for different values of $\theta$ i.e. $\theta=0^{\circ}, 30^{\circ}, 60^{\circ}$. From these graphs authors conclude that frequency for both the modes of vibration continuously increases with $\beta$. It is interesting to note that frequency increases as $\theta$ increases from $0^{\circ}$ to $60^{\circ}$.

Graphs (3) and (4) are drawn for the first and second mode of frequency respectively with $\beta$ varying from 0 to 1 for $\alpha_{1}=0, \mathrm{a} / \mathrm{b}=1.5, \alpha=0.4$ for different values of $\theta$ i.e. $\theta=0^{\circ}, 30^{\circ}, 60^{\circ}$. On comparing graphs $3 \& 4$ with graphs $1 \&$ 2 , authors found that the values of frequency for both the modes of vibration decrease as thermal gradient increases from 0.0 to 0.4 .
Graphs (5) and (6) represent the frequency for first two modes of vibration respectively for $\alpha=0, \beta=0, a / b=1.5$ at different values of $\theta$ i.e. $\theta=0^{\circ}, 30^{\circ}, 60^{\circ}$ for increasing values of $\alpha_{1}$ from 0.0 to 1.0. Again, it is interesting to see that frequency increases continuously with increases $\alpha_{1}$. Also frequency increases with increment in $\theta$.

Graph (7), (8), (9), (10) are plotted for both the modes of vibrations against increasing aspect ratio from 0.5 to 2.5 for the following cases:

Case (i) $\alpha=\beta=\alpha_{1}=0.0$ Graph (7) (for mode I) Graph (8) (for mode II)

Case (ii) $\alpha=\beta=\alpha_{1}=0.4$ Graph (9) (for mode I) Graph (10) (for mode II)

Frequency in these graphs is continuously increasing as $\mathrm{a} / \mathrm{b}$ increases from 0.5 to 2.5 but the frequency has less value in case (i) as compared to the case (ii).

\section{Comparison and Conclusion}

Special Case: $\alpha_{1}=\beta=0.0, \theta=0^{\circ}$

In this case, present plate will act as homogeneous and uniform clamped rectangular plate [3]. The results obtained

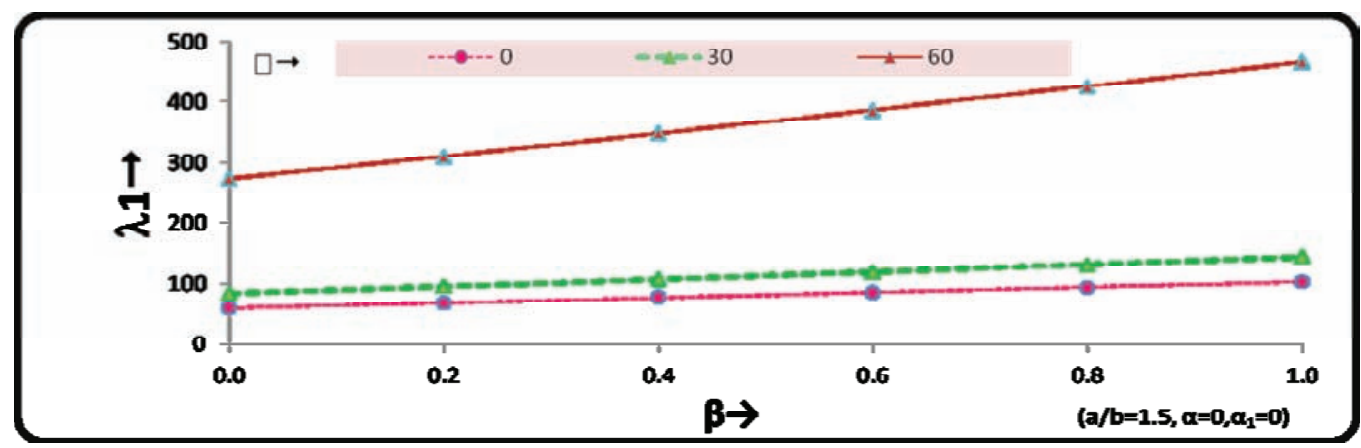

Graph 1. Frequency (Mode 1) vs Taper constant.

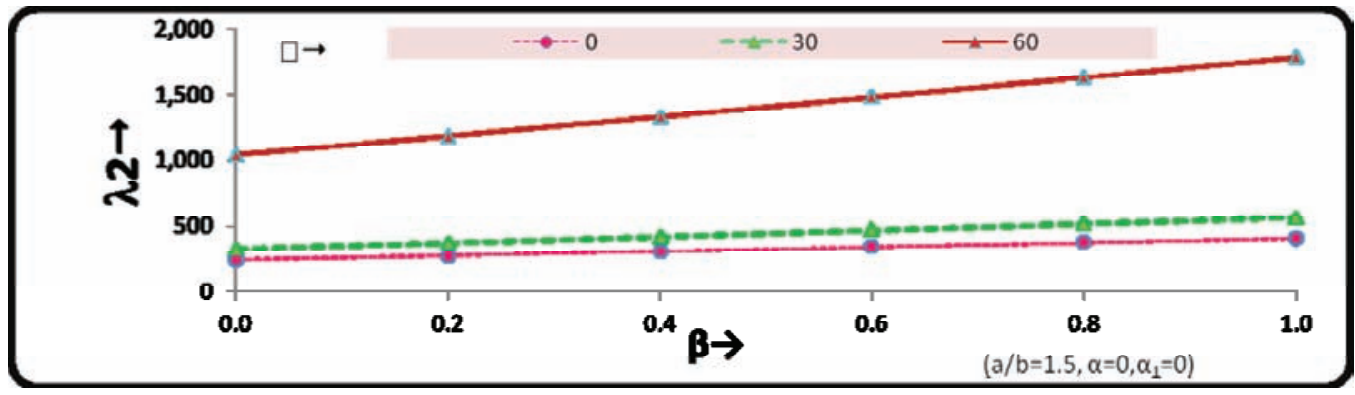

Graph 2. Frequency (Mode 2) vs Taper constant. 
Effect of Sinusoidal Thickness Variation on Vibrations of Non-homogeneous Parallelogram Plate with Bi-linearly Temperature Variations

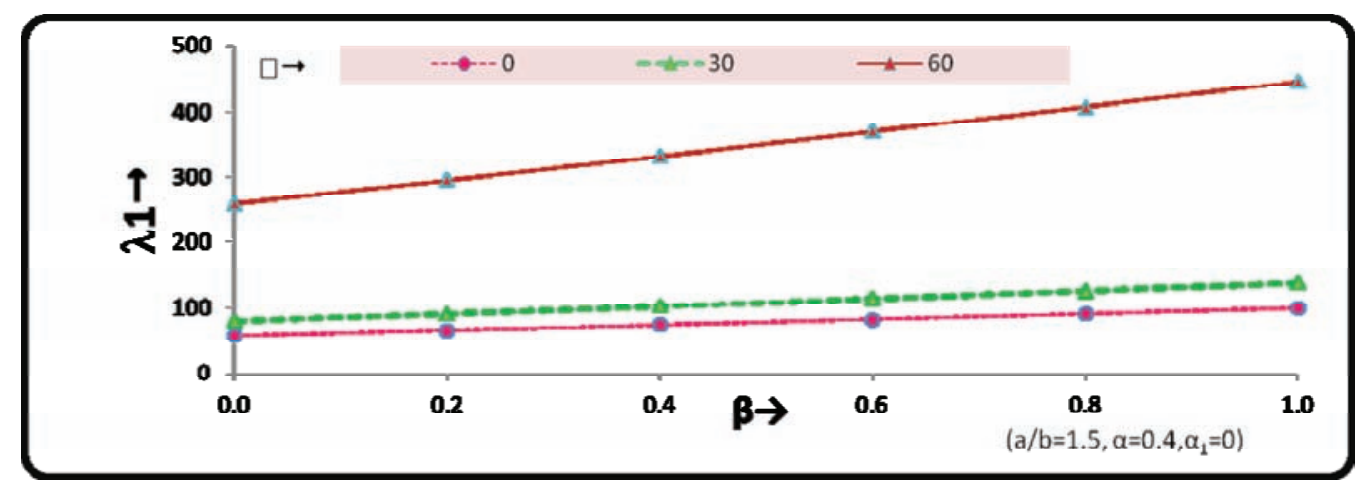

Graph 3. Frequency (Mode 1) vs Taper constant.

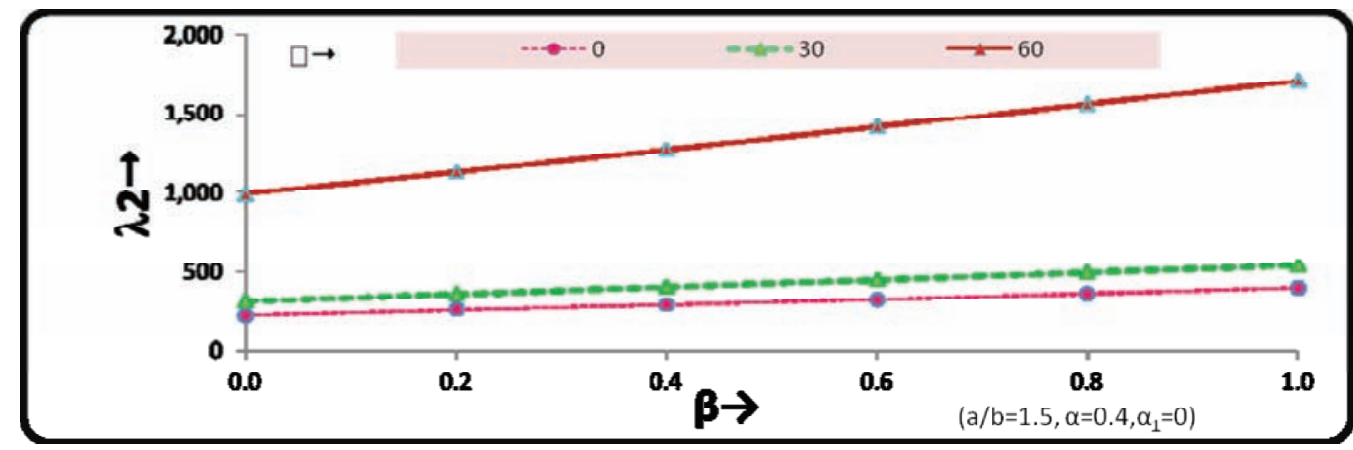

Graph 4. Frequency (Mode 2) vs Taper constant.

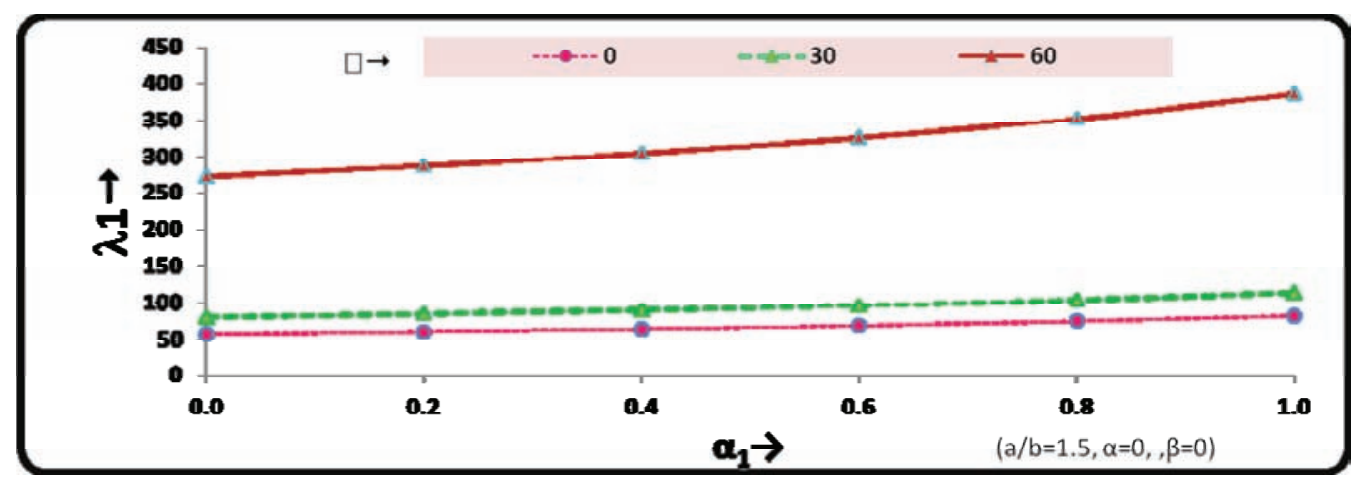

Graph 5. Frequency( Mode 1) vs Non-homogeneity constant.

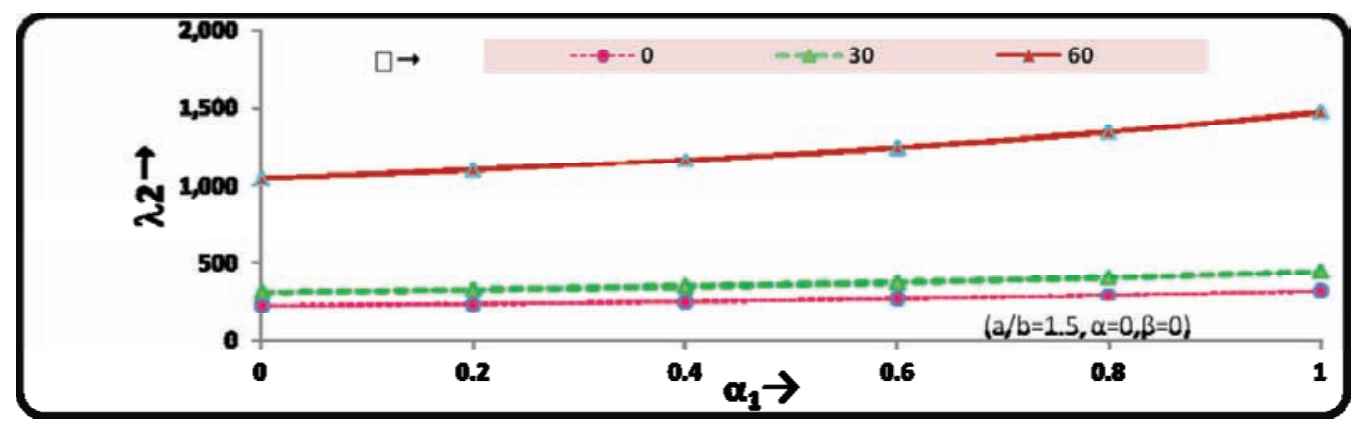

Graph 6. Frequency (Mode2) vs Non-homogeneity constant. 


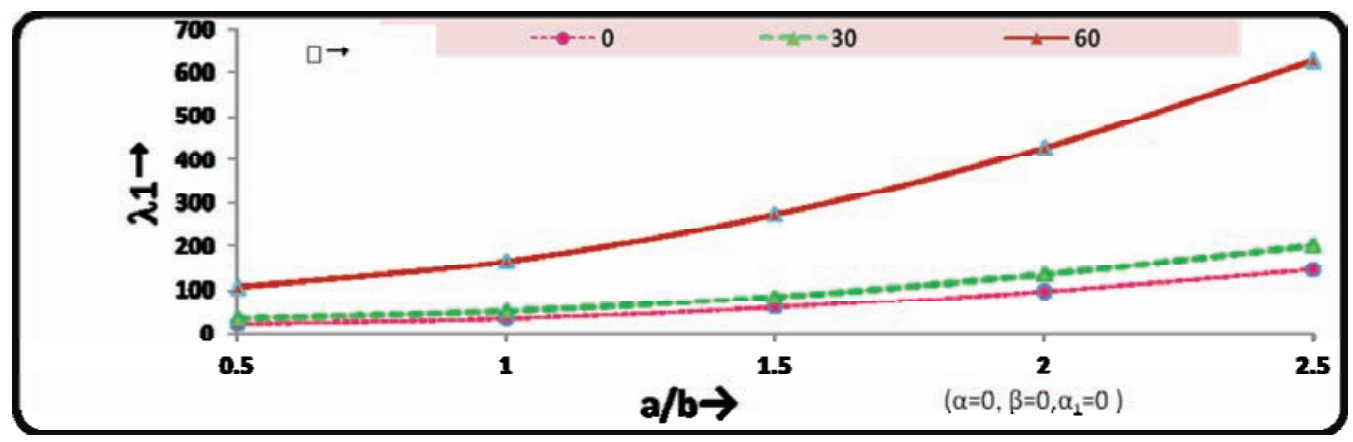

Graph 7. Frequency(Mode 1) vs Aspect Ratio.

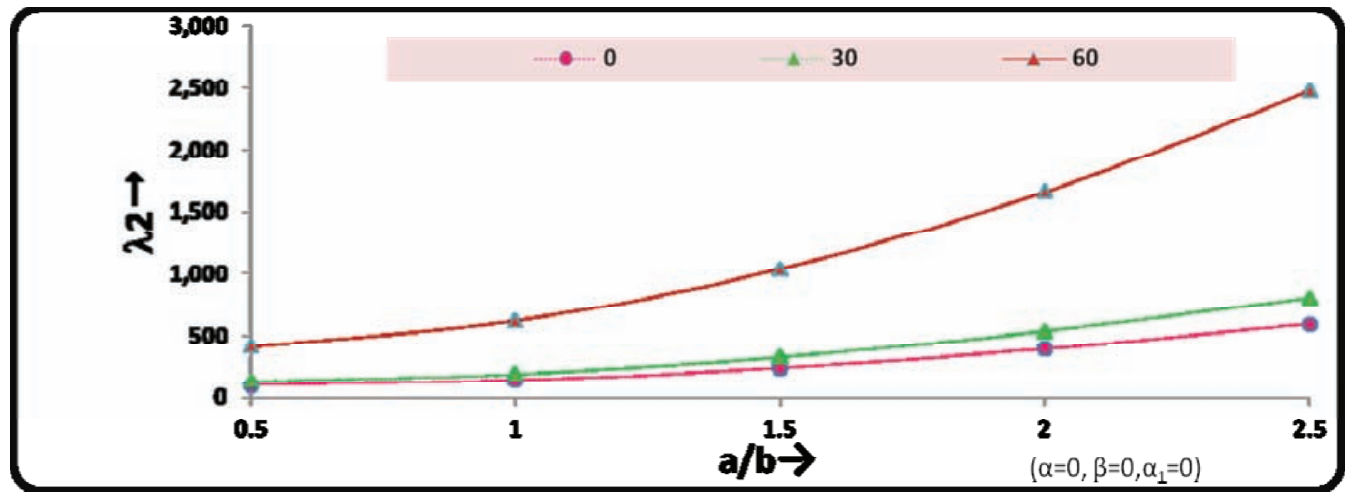

Graph 8. Frequency (Mode2) vs Aspect Ratio.

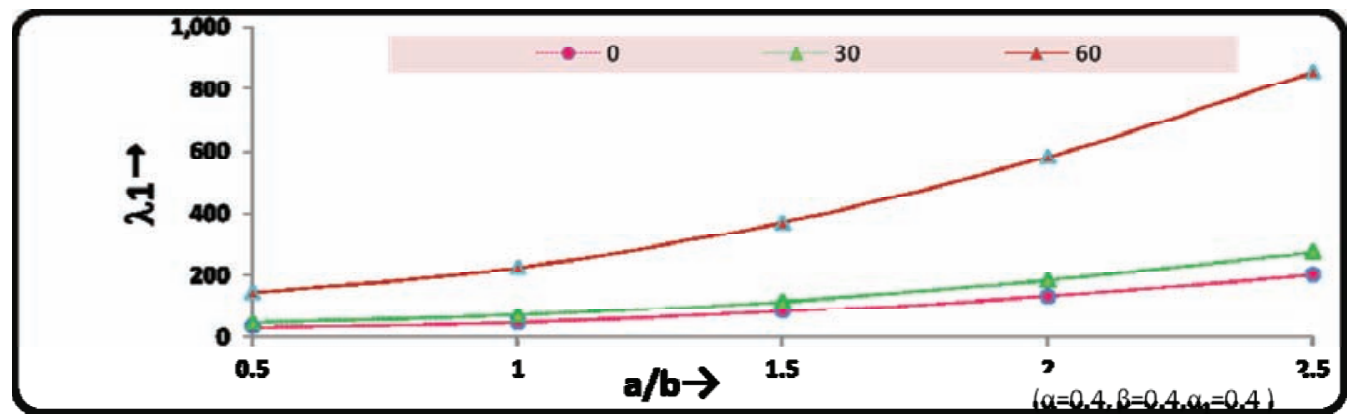

Graph 9. Frequency (Mode 1) vs Aspect Ratio.

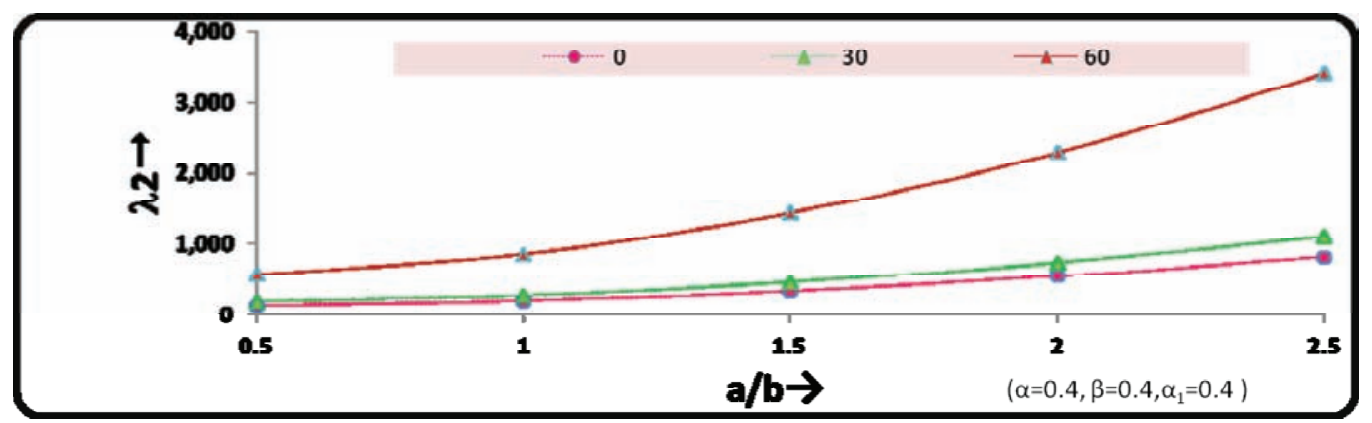

Graph 10. Frequency (Mode 2) vs Aspect Ratio. 
in this special case are compared with [3] \& found to be in close agreement.

A comparison is also made to the plate with [6] as:

\begin{tabular}{lll}
\hline $\boldsymbol{\theta}=30^{\circ}, \boldsymbol{\beta}=\mathbf{0 . 6}, \boldsymbol{\alpha}=\mathbf{0 . 4}, \boldsymbol{\alpha}_{\mathbf{1}}=\mathbf{0 , a} \mathbf{b}=\mathbf{1 . 0}$ \\
\hline & Mode I & Mode II \\
\hline Present Paper & 68.1 & 263.7 \\
REF [6] & 61.57 & 237.58 \\
\hline
\end{tabular}

It is found that there is approximately $11 \%$ increase in the frequency of the vibrations in the present paper as compared to [6]. Therefore, engineers and technocrats are advised to go through the findings of the present paper before finalizing any design of a machine or mechanical equipments so that they can produce more effective and reliable structures.

\section{References}

1. Singh B, and Sexena V (1997). Transverse vibration of skew plates with variable thickness, Journal of Sound and Vibration, vol 206, No.1, 1-13.

2. Chakarverty S, and Petyt M (1997). Natural frequencies for free vibration of non-homogeneous elliptic and circular plate using two dimensional orthogonal polynomials, Applied Mathematical Modelling, vol 21(7), 399-417.

3. Gupta A K, and Khanna A (2007). Vibration of visco-elastic rectangular plate with linearly varying thickness vibration in both directions, Journal of Sound and Vibration, vol 301, No 3-5, 450-457.

4. Gupta A K, and Khanna A (2010). Thermal effect on vibrations of parallelogram plate of linearly varying thickness, Advanced Studies of Theoretical Physics, vol 4, No.17, 817-826.

5. Gupta A K, Kumar A et al. (2010). Vibration of viscoelastic parallelogram plate with parabolic thickness variation, Applied Mathematics, vol 1, 128-136.
6. Gupta A K, and Kumar M (2011). Thermal effect of vibration of a parallelogram plate of bi-direction linearly varying thickness, Applied Mathematics, vol 2, 33-38.

7. Gupta A K, and Kaur H (2008). Study of the effect of thermal gradient on free vibration of clamped visco-elastic rectangular plates with linearly thickness variation in both directions, Meccanica, vol 43(4), 449-458.

8. Anupam K, and Sharma A K (2011). Thermally induced vibration of non-homogeneous visco-elastic plate of variable thickness, Advances in Physics, Theories and Application, vol $1,1-5$.

9. Khanna A, Kaur N et al. (2012). Effect of varying poisson ratio on thermally induced vibration of non-homogeneous rectangular plate, Indian Journal of Science and Technology, vol 5(9), 3263-3276.

10. Khanna A, and Sharma N (2012). Vibrational analysis of non-homogeneous tapered square plates with bi-parabolic temperature variations, International Journal of Mathematical Sciences and Applications, vol 6, No.VI, 327-334.

11. Lal R, and Dhanpati (2009). Effect of non-homogeneity on the vibration of orthotropic rectangular plates of varying thickness resting on a Pasternak foundation, Journal of Vibration and Acoustics: ASME, vol 131(1), $1-9$.

12. Leissa A W (1969). Vibration of plates, NASA SP, Scientific and Technical Information Division, National Aeronautics and Space Administration, vol 160.

13. Leissa A W (1987). Recent studies in plate vibration 19811985, Part-II complicating effect, The Shock and Vibration Digest, vol 19, 10-24.

14. Li W L (2004). Vibration analysis of rectangular plate with generalelasticboundarysupport,JournalofSoundandVibration, vol 273(3), 619-635.

15. Tomar J S, and Gupta A K (1983). Thermal effect on frequencies of an orthotropic rectangular plate of linearly varying thickness, Journal of Sound and Vibration, vol 90(3), 325-331. 